\title{
Hepatic $\beta$-arrestin 2 is essential for maintaining euglycemia
}

\author{
Lu Zhu, ${ }^{1}$ Mario Rossi, ${ }^{1}$ Yinghong Cui, ${ }^{1}$ Regina J. Lee,, ${ }^{1}$ Wataru Sakamoto, ${ }^{1}$ Nicole A. Perry, ${ }^{2}$ Nikhil M. Urs, ${ }^{3}$ Marc G. Caron, ${ }^{3}$ \\ Vsevolod V. Gurevich, ${ }^{2}$ Grzegorz Godlewski, ${ }^{4}$ George Kunos, ${ }^{4}$ Minyong Chen, ${ }^{5}$ Wei Chen, ${ }^{5}$ and Jürgen Wess ${ }^{1}$
}

'Molecular Signaling Section, Laboratory of Bioorganic Chemistry, National Institute of Diabetes and Digestive and Kidney Diseases (NIDDK), Bethesda, Maryland, USA. ${ }^{2}$ Department of Pharmacology, Vanderbilt University, Nashville, Tennessee, USA. ${ }^{3}$ Department of Cell Biology, Duke University Medical Center, Durham, North Carolina, USA. ${ }^{4}$ Laboratory of Physiologic Studies, National Institute on Alcohol Abuse and Alcoholism, Bethesda, Maryland, USA. 5Department of Medicine, Duke University Medical Center, Durham, North Carolina, USA.

\begin{abstract}
An increase in hepatic glucose production (HGP) represents a key feature of type 2 diabetes. This deficiency in metabolic control of glucose production critically depends on enhanced signaling through hepatic glucagon receptors (GCGRs). Here, we have demonstrated that selective inactivation of the GPCR-associated protein $\beta$-arrestin 2 in hepatocytes of adult mice results in greatly increased hepatic GCCR signaling, leading to striking deficits in glucose homeostasis. However, hepatocyte-specific $\boldsymbol{\beta}$-arrestin 2 deficiency did not affect hepatic insulin sensitivity or $\beta$-adrenergic signaling. Adult mice lacking $\beta$-arrestin 1 selectively in hepatocytes did not show any changes in glucose homeostasis. Importantly, hepatocyte-specific overexpression of $\beta$-arrestin 2 greatly reduced hepatic GCCR signaling and protected mice against the metabolic deficits caused by the consumption of a high-fat diet. Our data support the concept that strategies aimed at enhancing hepatic $\beta$-arrestin 2 activity could prove useful for suppressing HGP for therapeutic purposes.
\end{abstract}

\section{Introduction}

One key pathophysiological feature of type 2 diabetes (T2D) is an increase in hepatic glucose production (HGP) (1-3). HGP is modulated by the activity of several GPCRs, including hepatic glucagon receptors (GCGRs), which play a key role in maintaining euglycemia (3-5). The GCGR, which is abundantly expressed in hepatocytes, is coupled to the stimulatory $G$ protein $G$, which triggers pronounced increases in HGP through a pathway that involves cAMP-mediated activation of PKA $(5,6)$. Importantly, glucagon levels are inappropriately elevated in patients suffering from $\mathrm{T} 2 \mathrm{D}$, suggesting that enhanced GCGR activity may play a central role in the pathophysiology of T2D (3-7).

The activity of almost all GPCRs is modulated by a pair of proteins known as $\beta$-arrestin 1 and 2 (also known as arrestin 2 and 3 , respectively; ref. 8). $\beta$-Arrestins promote GPCR desensitization and internalization (8), but can also act as signaling proteins in their own right (9).

At present, it remains unknown whether hepatic GCGR activity is regulated by $\beta$-arrestins in vivo and, if yes, whether this regulation is physiologically relevant. To address this issue, we generated and analyzed mutant mice that lack $\beta$-arrestin 2 (Arrb2) or $\beta$-arrestin 1 (Arrb1) selectively in hepatocytes under the control of the thyroxine-binding globulin (TBG) promoter (Arrb2 $2^{f l f l}$ TBG-Cre and Arrb1 $1^{f / f l}$ TBG-Cre mice, referred to hereafter as hep-barr2-KO and hep-barr1-KO mice, respectively).

Authorship note: L. Zhu and M. Rossi contributed equally to this work. Conflict of interest: The authors have declared that no conflict of interest exists. Submitted: January 18, 2017; Accepted: May 11, 2017.

Reference information: J Clin Invest. 2017;127(8):2941-2945.

https://doi.org/10.1172/JCI92913.
Our findings indicate that hepatic $\beta$-arrestin 2 is critical for maintaining euglycemia by acting as a potent negative regulator of hepatic GCGR signaling in vivo.

\section{Results and Discussion}

Generation of hep-barr2-KO mice. To generate hep-barr2-KO mice, we injected floxed Arrb2 mice (10) via the tail vein with an adeno-associated virus (AAV) coding for Cre recombinase (AAVTBG-Cre) (11). For control purposes, we injected another set of floxed Arrb2 mice with the AAV-TBG-EGFP virus (11), which codes for EGFP (control mice). Two weeks after virus administration, we monitored Arrb2 and Arrb1 expression levels using RNA prepared from primary hepatocytes (Supplemental Figure 1, A and C; supplemental material available online with this article; https://doi.org/10.1172/JCI92913DS1).

Treatment of floxed Arrb2 mice with the AAV-TBG-Cre virus resulted in a pronounced and selective reduction of Arrb2 mRNA expression in hepatocytes (Supplemental Figure 1A). Moreover, $\beta$-arrestin 2 protein expression was no longer detectable in hepatocytes from these mice (Supplemental Figure 1B). Arrb2 deletion in hepatocytes had no significant effect on hepatic Arrb1 expression levels (Supplemental Figure 1C). Thus, we refer to floxed Arrb2 mice treated with the AAV-TBG-Cre virus as hep-barr2-KO mice throughout this study.

Hep-barr2-KO mice appeared healthy and had whole body and liver weights similar to those of their corresponding control mice (Supplemental Table 1). Moreover, livers from hep-barr2KO mice displayed no obvious morphological deficits (Supplemental Figure 1D).

Hepatic insulin action is not impaired in hep-barr2-KO mice. In a previous study, Luan et al. (12) proposed that $\beta$-arrestin 2 is required 
A

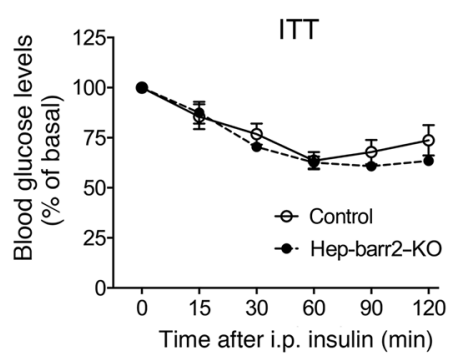

D

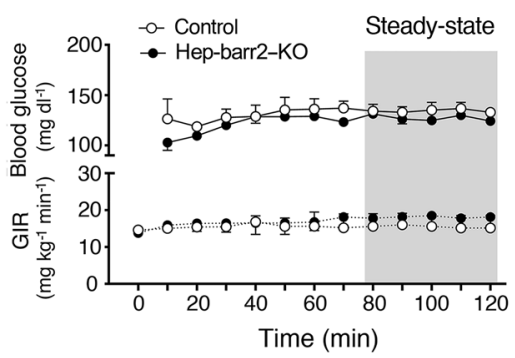

$\mathbf{E}$

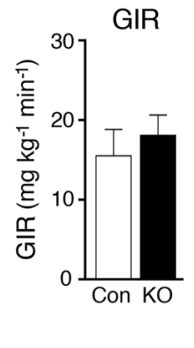

Figure 1. Insulin signaling is not impaired in hep-barr2-KO mice. (A) i.p. ITT. Data are shown as mean \pm SEM ( $n=9$ mice per group, 20-week-old males). (B) Insulin-induced phosphorylation of AKT and CSK $3 \alpha / \beta$ remains unaffected by the lack of $\beta$-arrestin 2 in hepatocytes. Primary hepatocytes prepared from hep-barr2-KO and control mice were incubated with insulin (10 nM) or saline for 15 minutes. Cell lysates were used for immunoblotting with the indicated antibodies. Representative blots are shown. See complete unedited blots in the supplemental material. (C) Quantification via densitometry (NIH Image) software) of the immunoblotting data shown in B. Phospho-protein expression levels were normalized by total AKT or total CSK3 $\alpha / \beta$ expression, respectively. Data represent mean \pm SEM ( $n=5$ mice per group, 16 - to 20 -week-old males). (D and E). Hyperinsulinemic euglycemic clamp studies. In $\mathbf{D}$, the time course of blood glucose and GIR are shown. Data in panel $\mathbf{E}$ were obtained during the steady-state period of the clamp (gray area in $\mathbf{D}$ ). Values are shown as mean \pm SEM ( $n=3$ or 4 mice per group, 20-week-old males). for proper insulin signaling in hepatocytes. In contrast, we found that hep-barr2-KO mice showed normal insulin sensitivity in an i.p. insulin tolerance test (ITT) (Figure 1A) and that insulin-stimulated phosphorylation of AKT and GSK3 remained unaffected by the lack of hepatic $\beta$-arrestin 2 (Figure 1, B and C). Finally, hyperinsulinemic euglycemic clamp studies did not reveal any significant changes in steady-state glucose infusion rates (GIR) between hepbarr2-KO and control mice (Figure 1, D and E).

These new findings clearly indicate that the lack of $\beta$-arrestin 2 in hepatocytes has no significant effect on insulin sensitivity. One possible reason for the discrepant findings reported by us (this study) versus Luan et al. (12) is that the previous study examined whole body Arrb2-KO mice. Since $\beta$-arrestin 2 is expressed in most tissues and cell types (8), hepatic insulin signaling may have been affected indirectly by $\beta$-arrestin 2 deficiency in other tissues.

$\beta$-Arrestin 2 acts as a potent negative regulator of GCGR signaling. In vivo studies showed that blood glucose levels were significantly increased in both fed and fasted hep-barr2-KO mice (Supplemental Table 1). Plasma insulin and glucagon levels were not significantly different between hep-barr2-KO and control mice (Supplemental Table 1).

Interestingly, following an acute dose of i.p. glucose, hepbarr2-KO mice showed significantly impaired glucose tolerance (Figure 2A) without significant changes in plasma insulin, glucagon, and glucagon-like peptide 1 (GLP-1) levels (Supplemental Figure 2). To test the hypothesis that hepatic $\beta$-arrestin 2 deficiency may lead to enhanced hepatic GCGR signaling, we treated mice with a single i.p. dose of an anti-GCGR mAb (mAb7.v44; 10 $\mathrm{mg} / \mathrm{kg}$ ). In control experiments, this Ab completely prevented glu- cagon-induced (16 $\mu \mathrm{g} / \mathrm{kg}$ i.p.) increases in blood glucose levels in WT C57BL/6 mice (Supplemental Figure 3). Remarkably, following treatment of hep-barr2-KO mice with the anti-GCGR Ab, the mutant mice no longer displayed impaired glucose tolerance (Figure 2, A and B). This observation suggests that the impairment in glucose tolerance caused by hepatic $\beta$-arrestin 2 deficiency is probably due to enhanced hepatic GCGR signaling. The ability of the anti-GCGR Ab to improve glucose tolerance in glucose-injected control mice (compare Figure 2, A and B) also clearly indicates that GCGR signaling regulates postprandial blood glucose levels.

We obtained very similar results when we treated hep-barr2$\mathrm{KO}$ and control mice with the gluconeogenic substrate pyruvate (pyruvate tolerance test; Figure 2, C and D). Moreover, following an acute dose of i.p. glucagon $(16 \mu \mathrm{g} / \mathrm{kg})$, hep-barr2-KO mice showed greatly enhanced blood glucose excursions (glucagon challenge test; Figure 2E).

Hepatic glycogen and triglyceride levels were significantly reduced in livers from hep-barr2-KO mice (Figure 2, J and $\mathrm{K}$ ). Since glucagon stimulates hepatic glycogen breakdown and fatty acid oxidation and inhibits insulin-dependent lipogenesis $(5,13)$, this observation further supports the notion that GCGR signaling is enhanced in $\beta$-arrestin 2-deficient hepatocytes.

cAMP assays showed that glucagon-induced increases in cAMP levels were significantly enhanced in hepatocytes lacking $\beta$-arrestin 2 (Figure 2F). We obtained similar results when we knocked down the expression of Arrb2 in primary WT mouse hepatocytes by using Arrb2 siRNA (Supplemental Figure 4). In this latter case, we monitored the activity of the cAMP/PKA signaling pathway by using a CRE-luciferase reporter. 
A
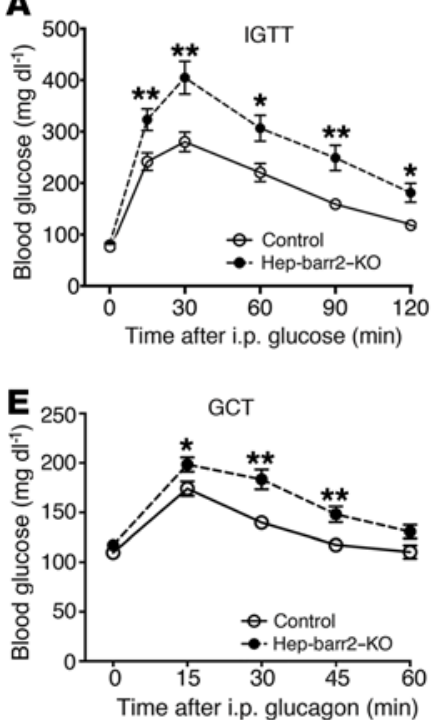

B

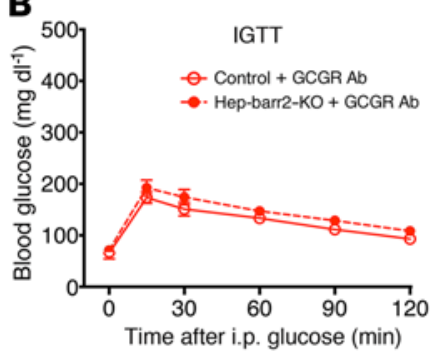

$\mathbf{F}$

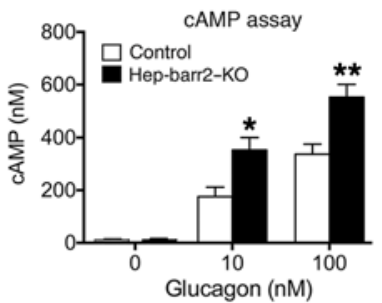

C

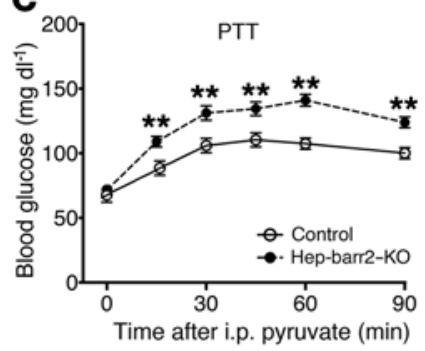

G

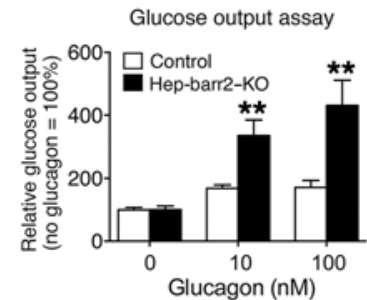

D

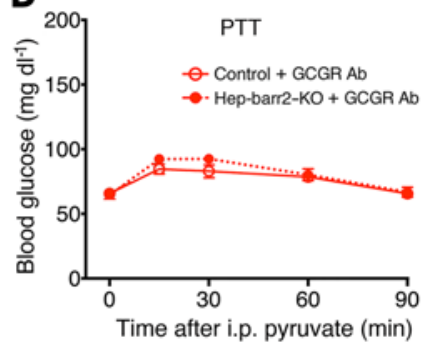

H
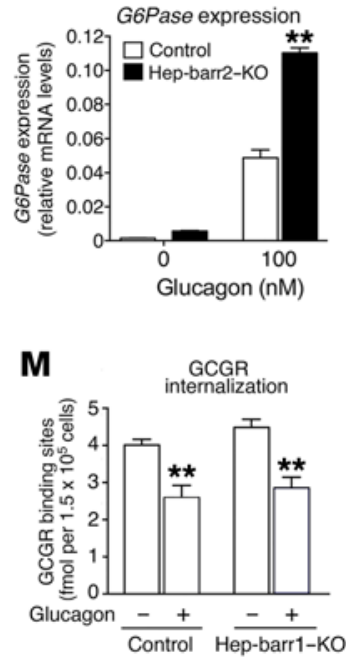
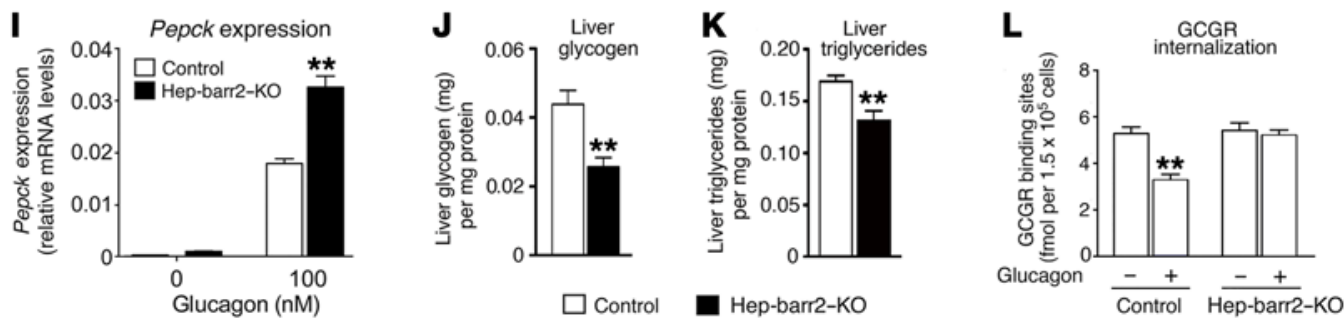

Figure 2. Metabolic phenotyping and GCGR internalization studies. (A and B) i.p. Glucose tolerance tests (ICTT). (C and D) i.p. pyruvate tolerance tests (PTT). In B and D, an anti-GCGR Ab (10 mg/kg i.p) was administered approximately 24 hours prior to glucose or pyruvate injections, respectively. (E) i.p. glucagon challenge test (CCT). (F-I) In vitro studies with primary mouse hepatocytes. The ability of glucagon to stimulate intracellular cAMP accumulation (F), glucose production (G), and the expression of the G6Pase (H) and Pepck (I) genes was studied using primary hepatocytes prepared from hep-barr2-KO mice and their control littermates. Gene expression data were normalized relative to the expression of $\beta$-actin (quantitative reverse-transcriptase PCR [qRT-PCR] analysis). Hepatocyte data are shown as mean \pm SEM of at least 3 independent experiments. (J and $\mathbf{K}$ ) Hepatic glycogen and triglyceride content. All studies were carried out with male mice consuming RC (mouse age, 11-20 weeks). Data represent mean $\pm \mathrm{SEM}\left(n=7-10\right.$ mice per group) (A-K). ${ }^{*} P<0.05$; ${ }^{*} P<0.01$, versus control (Student's $t$ test). (L and M) GCGR internalization assays. Primary hepatocytes prepared from hep-barr2-KO (L) or hep-barr1-KO (M) mice and their control littermates were stimulated with glucagon (100 nM) for 30 minutes at $37^{\circ} \mathrm{C}$. Cell surface GCCRs were labeled with I $\mathrm{I}^{125}$-glucagon. Data represent the mean \pm SEM of 3 independent experiments, each carried out in triplicate. ${ }^{*} P<0.01$, versus cells not exposed to glucagon (Student's $t$ test).

We also found that glucagon-induced glucose output was greatly enhanced in $\beta$-arrestin 2-deficient hepatocytes (Figure $2 \mathrm{G}$ ). Consistent with this observation, hepatic $\beta$-arrestin 2 deficiency led to significant increases in glucagon-stimulated glucose-6-phosphatase catalytic subunit (G6pase) and phosphoenolpyruvate carboxykinase (Pepck) transcript levels (G6pase and PEPCK are the 2 rate-limiting enzymes of gluconeogenesis) (Figure 2, $\mathrm{H}$ and I).

$\mathrm{I}^{125}$-glucagon radioligand binding studies with primary hepatocytes showed that the lack of $\beta$-arrestin 2 had no significant effect on GCGR densities (receptor numbers per cell: control, 2.19 $\pm 0.12 \times 10^{4}$; hep-barr2-KO, $\left.2.25 \pm 0.13 \times 10^{4}\right)$. In summary, these data strongly support the concept that $\beta$-arrestin 2 represents a potent suppressor of hepatic GCGR signaling in vivo and in vitro.

hep-barr1-KO mice show normal glucose homeostasis. Like most cell types, mouse hepatocytes express both $\beta$-arrestin 2 and 1 (12). By employing the same strategy as described above for the generation of hep-barr2-KO (10), we used floxed Arrb1 mice (J. Kim and R.J. Lefkowitz, unpublished observations) to obtain mutant mice that selectively lacked $\beta$-arrestin 1 in hepatocytes (hep-barr1-KO mice)
(Supplemental Figure 5, A and B). The lack of $\beta$-arrestin 1 had no significant effect on GCGR expression in primary hepatocytes (receptor number per cell: control, $1.66 \pm 0.06 \times 10^{4}$; hep-barr1-KO, 1.86 $\left.\pm 0.08 \times 10^{4}\right)$, as determined in $\mathrm{I}^{125}$-glucagon binding studies. Blood glucose, plasma insulin, and plasma glucagon levels remained unaffected by hepatic $\beta$-arrestin 1 deficiency (Supplemental Table 2). Moreover, and in striking contrast with the metabolic deficits displayed by the hep-barr2-KO mice, hep-barr1-KO mice did not show any impairments in glucose homeostasis (Supplemental Figure 6).

GCGR internalization is abolished in hepatocytes lacking $\beta$-arrestin 2. To examine whether $\beta$-arrestin 2 is required for the internalization of endogenous GCGRs, we used $\mathrm{I}^{125}$-glucagon to assess the density of GCGRs present on the surface of primary hepatocytes prepared from hep-barr2-KO or control mice (Figure 2L). Prior to glucagon treatment, cell surface GCGR densities were similar in $\mathrm{KO}$ and control hepatocytes (Figure 2L). Glucagon $(100 \mathrm{nM})$ treatment of control hepatocytes led to the internalization of approximately $35 \%$ of cell surface GCGRs (Figure 2L). In striking contrast, glucagon failed to induce GCGR internaliza- 

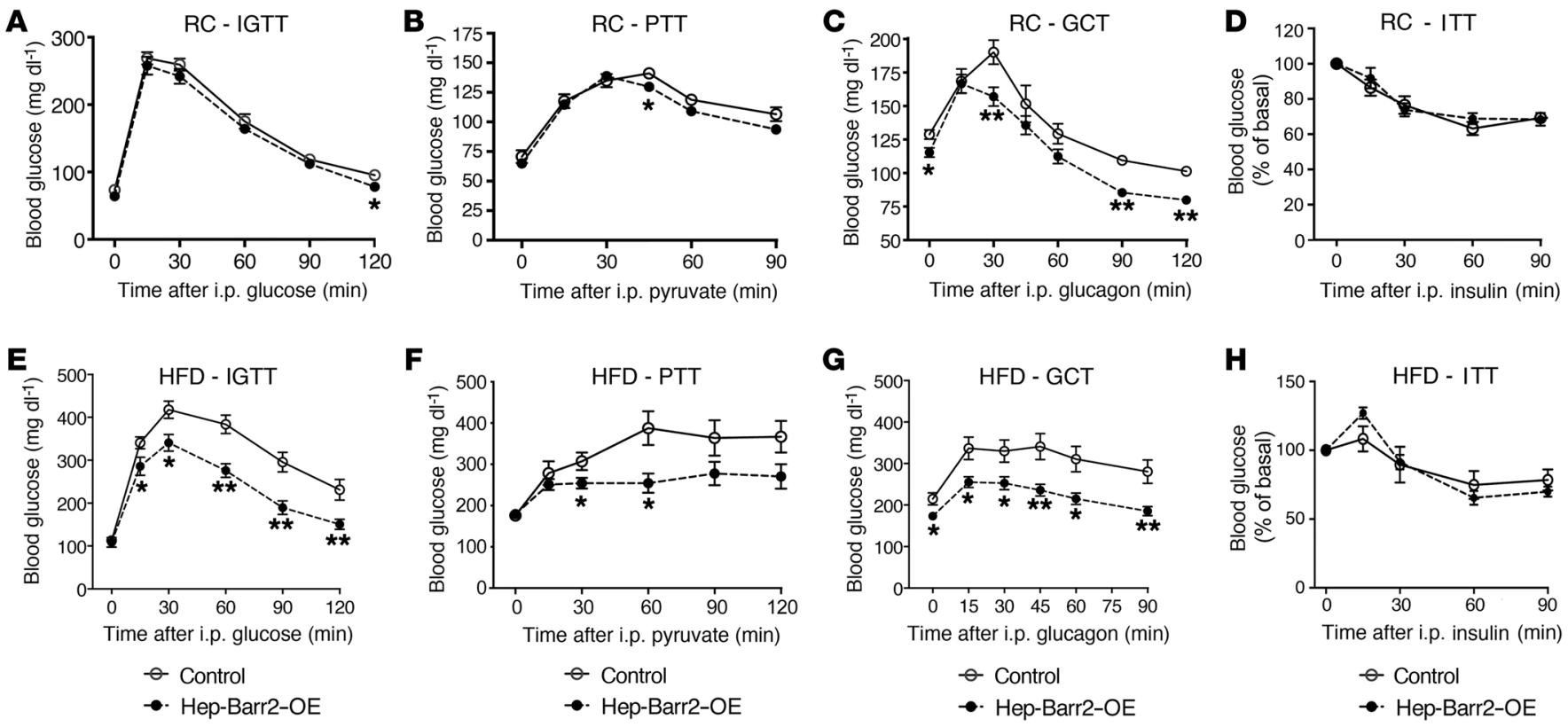

Figure 3. In vivo studies with hep-barr2-OE mice and their control littermates. (A-H) In vivo metabolic tests carried out with mice maintained on RC (A-D) or HFD (E-H). Animals were maintained on the HFD for at least 8 weeks. Mice were subjected to i.p. glucose tolerance test (A and $\mathbf{E})$, pyruvate tolerance test (B and $\mathbf{F}$ ), glucagon challenge test ( $\mathbf{C}$ and $\mathbf{G}$ ), and ITT ( $\mathbf{D}$ and $\mathbf{H})$. Data are shown as mean \pm SEM (8-10 mice per group; 12- to 16-week-old males). ${ }^{*} P<0.05 ;{ }^{* *} P<0.01$, versus control (Student's $t$ test).

tion in $\beta$-arrestin 2-deficient hepatocytes (Figure 2L). Additional $\mathrm{I}^{125}$-glucagon-binding studies showed that glucagon-induced GCGR internalization remained intact in hepatocytes lacking $\beta$-arrestin 1 (Figure 2M), indicating that hepatic GCGR internalization is mediated by $\beta$-arrestin 2 . Since receptor internalization contributes to GPCR desensitization (8), lack of GCGR internalization in $\beta$-arrestin 2 -deficient hepatocytes is predicted to play a role in enhanced glucagon signaling.

Interestingly, bioluminescence resonance energy transfer (BRET) studies carried out with cotransfected COS-7 cells clearly demonstrated that activated GCGRs can interact with both $\beta$-arrestins (tagged with a Venus reporter; Supplemental Figure 7). Since $\beta$-arrestin 1 recruitment did not interfere with GCGR internalization (Figure 2M), it is likely that activated GCGRs induce different conformational changes in $\beta$-arrestin 2 versus $\beta$-arrestin 1. This phenomenon of conformational flexibility is currently the focus of many laboratories studying $\beta$-arrestin function (14).

Lack of $\beta$-arrestins has little effect on $\beta$-adrenergic receptor signaling in hepatocytes. Treatment of hepatocytes from control and hep-barr2-KO (and hep-barr1-KO) mice with isoproterenol (Iso), a $\beta$-adrenergic receptor agonist ( 1 and $10 \mu \mathrm{M})$, caused only small increases in cAMP accumulation (compared with glucagon) and had no significant effect on glucose release (Supplemental Figures 8 and 9). Following Iso $(10 \mu \mathrm{M})$ treatment, hep-barr2-KO hepatocytes (Supplemental Figure 8A), but not hep-barr1-KO hepatocytes (Supplemental Figure 9A), showed a small but significant increase in intracellular cAMP levels. Iso was unable to stimulate glucose release in control or mutant hepatocytes (Supplemental Figure 8B and Supplemental Figure 9B). It is therefore unlikely that altered $\beta$-adrenergic receptor signaling makes a major contribution to the metabolic phenotypes observed in the hep-barr2-KO mice.
PTX treatment does not affect the increase in GCGR-mediated glucose output in hep-barr2-KO hepatocytes. To exclude a role of $\mathrm{G}_{\mathrm{i}}$ type proteins in the observed metabolic phenotypes, we monitored glucagon-induced $(10 \mathrm{nM})$ changes in cAMP accumulation and glucose release in hep-barr2-KO hepatocytes, either in the absence or presence of pertussis toxin (PTX) (300 ng/ml). In cAMP assays, glucagon-stimulated cAMP levels were significantly increased in PTX-treated control cells (Supplemental Figure 10A), suggesting that inactivation of inhibitory $G_{i}$-type $G$ proteins enhances GCGR signaling via $G_{s}$. This stimulatory effect of PTX was not observed with glucagon-treated hep-barr2-KO hepatocytes (Supplemental Figure 10A), probably due to the fact that cAMP levels were already maximally activated under these experimental conditions. The presence of PTX had no significant effect on glucagon-induced glucose responses in control and hep-barr2-KO hepatocytes (Supplemental Figure 10B), suggesting that it is unlikely that potential signaling of the GCGR via $G_{i}$ makes an important contribution to the changes in glucose homeostasis displayed by the hep-barr2-KO mice.

Overexpression of $\beta$-arrestin 2 in hepatocytes protects mice against obesity-induced metabolic deficits. To generate mice that overexpressed $\beta$-arrestin 2 selectively in hepatocytes (hep-barr2-OE mice), we injected WT C57BL/6 mice with an AAV coding for an HA-tagged version of $\beta$-arrestin 2 under the transcriptional control of the hepatocyte-selective $T B G$ promoter (Supplemental Figure 11, A and B). Overexpression of $\beta$-arrestin 2 in hepatocytes had no significant effect on Arrb1 expression levels (Supplemental Figure 11A).

Initially, we subjected hep-barr2-OE mice consuming regular chow (RC) and their control littermates (AAV-TBG-EGFP-injected WT C57BL/6 mice) to a series of in vivo metabolic studies. Hepbarr2-OE mice showed significant decreases in fed blood glucose levels (Supplemental Table 3). Plasma insulin and glucagon levels 
were similar in hep-barr2-OE mice and their control littermates (Supplemental Table 3). The hep-barr2-OE mice showed only very minor reductions in blood glucose excursions in glucose and pyruvate tolerance tests (Figure 3, A and B). Importantly, however, the mutant mice were clearly less sensitive to the hyperglycemic effects of glucagon in a glucagon challenge test (Figure 3C). Consistent with this in vivo phenotype, glucagon-stimulated HGP was significantly reduced in primary hepatocytes prepared from hep-barr2OE mice (Supplemental Figure 11C), further confirming that Arrb2 represents a potent negative regulator of hepatic GCGR function.

We performed similar metabolic tests with hep-barr2-OE mice and their control littermates maintained on a high-fat diet (HFD) for 6 to 10 weeks (Figure 3, E-H). Under these experimental conditions, the control mice showed significantly greater increases in blood glucose levels in all in vivo metabolic assays (as compared with RC control mice), indicative of glucose intolerance, increased gluconeogenesis, and enhanced glucagon sensitivity (Figure 3, E-G). Strikingly, these metabolic deficits were not observed with hep-barr2-OE mice maintained on the HFD (Figure 3, E-G). HFD hep-barr2-OE mice displayed significantly reduced fed and fasting blood glucose levels (Supplemental Table 3). Plasma insulin and glucagon levels were not significantly affected by hepatic $\beta$-arrestin 2 overexpression (Supplemental Table 3). Moreover, hep-barr2-OE and control mice showed similar glucose responses in an ITT (Figure 3, D and H).

Interestingly, hepatic Arrb2 expression levels were significantly lower in HFD control than in RC control mice (Supplemental Figure 12). Consistent with this observation, a recent study (12) reported that hepatic $\beta$-arrestin 2 levels are reduced in diabetic individuals. On the basis of these observations, reduced hepatic $\beta$-arrestin 2 activity may contribute to the pathophysiology of T2D.

Conclusions. In summary, we here demonstrate that hepatic $\beta$-arrestin 2 is critical for maintaining euglycemia. At the cellular level, $\beta$-arrestin 2 acts as a potent negative regulator of hepatic GCGR signaling in vivo and in vitro. Interestingly, hepatic $\beta$-arrestin 1 , in contrast with $\beta$-arrestin 2 , does not play a role in regulating hepatic GCGR activity and euglycemia, indicating that the $2 \beta$-arrestins do not have redundant functions in hepatocytes. Our findings suggest that strategies aimed at enhancing the expression or activity of hepatic $\beta$-arrestin 2 or the development of small molecule $\beta$-arrestin 2 mimetics may become clinically useful for reducing exaggerated hepatic GCGR signaling for the treatment of T2D $(3,6)$.

\section{Methods}

Detailed methods are described in Supplemental Methods. See complete unedited blots in the supplemental material (see also ref. 15).

Statistics. Data are expressed as mean \pm SEM for the indicated number of observations. The statistical tests used are indicated in the figure legends. All Student's $t$ tests used were 2 tailed. A $P$ value of less than 0.05 was considered significant.

Study approval. All animal studies were approved by the NIDDK Institutional Animal Care and Use Committee.

\section{Author contributions}

LZ, MR, VVG, GG, GK, and JW designed and conceived the experiments. LZ, MR, WS, YC, RJL, and NAP performed and analyzed experiments. NMU and MGC provided the floxed Arrb2 mice and offered valuable advice. MC and WC generated the floxed Arrb1 mice and provided critical know-how regarding the use of these mice. NAP and VVG carried out and interpreted the BRET experiments. GG and GK, together with LZ, performed and analyzed the clamp studies. LZ and JW wrote the manuscript.

\section{Acknowledgments}

This research was funded by the Intramural Research Program of the NIDDK (to LZ, MR, YC, WS, and JW) and the National Institute on Alcohol Abuse and Alcoholism (to GG and GK). Robert J. Lefkowitz and his colleagues at Duke University provided the floxed Arrb1 mice. Tina M. Iverson (Vanderbilt University) cosupervised the BRET experiments. MGC and WC were supported by NIH grants 5R37-MH-073853 and R01-CA172570, respectively. VVG was supported by NIH RO1 GM109955 and EY011500. MC was the recipient of a Clinical Oncology Research Center Development grant (5K12-CA100639-08).

Address correspondence to: Jürgen Wess, Laboratory of Bioorganic Chemistry, Molecular Signaling Section, National Institute of Diabetes and Digestive and Kidney Diseases, Building 8A, Room B1A-05, 8 Center Drive, Bethesda, Maryland 20892, USA. Phone: 301.402.3589; Email:jurgenw@niddk.nih.gov.

NMU's present address is: Department of Pharmacology \& Therapeutics, University of Florida, Gainesville, Florida, USA.
1. Postic C, Dentin R, Girard J. Role of the liver in the control of carbohydrate and lipid homeostasis. Diabetes Metab. 2004;30(5):398-408.

2. Lin HV, Accili D. Hormonal regulation of hepatic glucose production in health and disease. Cell Metab. 2011;14(1):9-19.

3. Unger RH, Cherrington AD. Glucagonocentric restructuring of diabetes: a pathophysiologic and therapeutic makeover.JClin Invest. 2012;122(1):4-12.

4. Jiang G, Zhang BB. Glucagon and regulation of glucose metabolism. Am J Physiol Endocrinol Metab. 2003;284(4):E671-E678.

5. Estall JL, Drucker DJ. Glucagon and glucagonlike peptide receptors as drug targets. Curr Pharm Des. 2006;12(14):1731-1750.

6. Cho YM, Merchant CE, Kieffer TJ. Targeting the glucagon receptor family for diabetes and obesity therapy. Pharmacol Ther. 2012;135(3):247-278.

7. D'Alessio D. The role of dysregulated glucagon secretion in type 2 diabetes. Diabetes Obes Metab. 2011;13 Suppl 1:126-132.

8. Pierce KL, Premont RT, Lefkowitz RJ. Seventransmembrane receptors. Nat Rev Mol Cell Biol. 2002;3(9):639-650.

9. Shukla AK, Xiao K, Lefkowitz RJ. Emerging paradigms of $\beta$-arrestin-dependent seven transmembrane receptor signaling. Trends Biochem Sci. 2011;36(9):457-469.

10. Urs NM, et al. Distinct cortical and striatal actions of a $\beta$-arrestin-biased dopamine $\mathrm{D} 2$ receptor ligand reveal unique antipsychotic-like properties. Proc Natl Acad Sci U S A. 2016;113(50):E8178-E8186.
11. Miller RA, et al. Adiponectin suppresses gluconeogenic gene expression in mouse hepatocytes independent of LKB1-AMPK signaling. J Clin Invest. 2011;121(6):2518-2528.

12. Luan B, et al. Deficiency of a beta-arrestin-2 signal complex contributes to insulin resistance. Nature. 2009;457(7233):1146-1149.

13. Rui L. Energy metabolism in the liver. Compr Physiol. 2014;4(1):177-197.

14. Gurevich VV, Gurevich EV. Extensive shape shifting underlies functional versatility of arrestins. Curr Opin Cell Biol. 2014;27:1-9.

15. Ruiz de Azua I, et al. Spinophilin as a novel regulator of $\mathrm{M} 3$ muscarinic receptor-mediated insulin release in vitro and in vivo. FASEB J. 2012;26(10):4275-4286. 